\title{
Antibiotic Prophylaxis Trials in Obstetrics: A Call for Pediatric Collaboration
}

\author{
Rodney A. McLaren, Jr., MD ${ }^{1}$ Fouad Atallah, MD ${ }^{1}$ \\ ${ }^{1}$ Department of Obstetrics and Gynecology, Maimonides Medical \\ Center, Brooklyn, New York \\ 2 Department of Obstetrics and Gynecology, SUNY Downstate \\ Medical Center, Brooklyn, New York
}

Howard Minkoff, $\mathrm{MD}^{1,2}$

Am J Perinatol Rep 2020;10:e155-e158.

\begin{abstract}
Address for correspondence Rodney A. McLaren, Jr., MD, Department of Obstetrics and Gynecology, Maimonides Medical Center, 967 48th Street, Brooklyn, NY 11219 (e-mail: rmclaren624@gmail.com).
\end{abstract}

\begin{abstract}
Keywords

- antibiotic prophylaxis

- cesarean delivery

- asthma

- childhood obesity

- microbiota

- neonatal microbiome

Surgical site infections are common complications of cesarean delivery. Many recent studies, including meta-analyses, have assessed the efficacy of antibiotic prophylaxis. Those articles have demonstrated that preincision antibiotic prophylaxis reduces the incidence of surgical site infections postcesarean, and that the use of adjunctive azithromycin further reduces infection after nonelective cesarean deliveries. However, long-term effects of fetal exposure to antibiotic prophylaxis-including asthma, obesity, and alterations in microbiota-have also been demonstrated. We suggest that while studies of optimal antibiotic regimens proceed, considerations of the potential risks to the neonate should be factored into discussions of benefits and burdens.
\end{abstract}

The prevalence of cesarean delivery in the United States has been increasing since the 1990s. ${ }^{1}$ Surgical site infections occur after approximately 5 to $12 \%$ of cesarean deliveries and are among the most common complications after the procedure. $^{2-5}$ There have been many well-designed studies that have assessed a variety of antibiotic prophylaxis regimens designed to reduce this complication. For example, a metaanalysis of three randomized clinical trials demonstrated that antibiotic prophylaxis given prior to skin incision rather than after umbilical cord clamping significantly reduced the incidence of surgical site infections. ${ }^{2}$ Recently, a randomized clinical trial of azithromycin added to the usual antibiotic prophylaxis regimen prior to nonelective cesarean deliveries demonstrated a significant reduction in infectious morbidity from 12 to $6.1 \%{ }^{6}$ Another meta-analysis of 16 trials demonstrated that women who had vaginal preparation prior to unscheduled cesarean delivery also had a significant reduction in endometritis compared with women who did not receive vaginal preparation. ${ }^{7}$ However, a subsequent secondary analysis of the adjunctive azithromycin trial demonstrated that vaginal preparation did not make a difference in the incidence of surgical site infections. ${ }^{8}$ Finally, Harper et al performed a cost analysis and demonstrated that adding azithromycin to the

received

December 29, 2019

accepted

March 5, 2020
DOI https://doi.org/

10.1055/s-0040-1709513. ISSN 2157-6998. usual antibiotic prophylaxis for both elective and nonelective cesarean deliveries would be cost saving. ${ }^{9}$ However, among these critical works, little attention is paid to the long-term effects of antibiotic prophylaxis exposure on the fetus. Costantine et al's meta-analysis of three randomized trials demonstrated no difference in neonatal sepsis or neonatal intensive care unit admissions, but did not include any longer neonatal follow-up. ${ }^{2}$ The randomized trial of the adjunctive azithromycin trial also had short neonatal follow-up that also showed no differences in readmissions at 3 months of age between the groups. ${ }^{6}$ We believe the failure to consider neonatal consequences represents an important knowledge gap.

Our argument is not that the hypothetical risks to neonates of antibiotic exposure should, a priori, outweigh the demonstrated benefits of antibiotics to women. Rather, we believe that nascent evidence pointing to potential risks to children from in utero exposure to antibiotics-described later-deserves the attention of researchers and academicians before they expose ever increasing numbers of children to these agents. Other disciplines of medicine have also raised concerns regarding the long-term effect of antibiotics on neonatal microbiome and development of chronic diseases such as obesity. ${ }^{10,11}$ Evidence of these concerns have
Copyright $\odot 2020$ by Thieme Medical Publishers, Inc., 333 Seventh Avenue, New York, NY 10001, USA. Tel: +1(212) 760-0888.
License terms

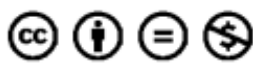


recently been expressed by several professional organizations. For example, the U.S. Preventive Services Task Force updated their guideline of routine urine culture testing among pregnant women from a Grade A to a Grade B recommendation based on recent understanding of the influence of antibiotics on the microbiome. ${ }^{12}$ In addition, the updated guideline on group B streptococcal (GBS) infections by the American Academy of Pediatrics highlighted the risks of disruption of the infant microbiome from prenatal antibiotic exposure and noted that the secondary effects of this intervention are unknown and an area of active investigation. ${ }^{13}$ Finally, one of the reasons for the postdelivery timing of intervention in the ANODE trial was concern about antibiotics on the infant microbiome. ${ }^{14}$ Thus, investigators should recognize the potential for risk, and include plans for follow-up of children, and should explore ways to mitigate possible adverse consequences. Up until now potential, and potentially serious, risks have been largely ignored in the design and interpretation of studies, and in their translation into treatment guidelines. Cost/benefit or risk/benefit analyses that underpin recommendations of therapeutic regimens may not be as useful if neonatal consequences are not part of the calculus.

\section{Evidence}

Culture-based studies, which have shown the presence of microbes in the placenta, amniotic fluid, fetal membrane, umbilical cord blood, and meconium, suggest that neonatal microbiome development may begin at birth. ${ }^{15}$ One published hypothesis is that the maternal gut microbiota may be transferred to the fetus via the bloodstream. ${ }^{16}$ Labeled bacterial species that were given to pregnant mice orally were found in the meconium of their offspring. ${ }^{16}$ Another exposure to microbes in the newborn is during the delivery, either by vaginal flora after vaginal delivery or by common skin flora after cesarean delivery. ${ }^{15}$ What is important for this discussion is that the neonatal microbiome can be disrupted by antibiotic exposure. Cox et al performed a series of experiments using low-dose penicillin that was given to pregnant mice and their offspring at different times (e.g., pregnant mice were given low-dose penicillin right before birth) and the microbiota was tested in the offspring. They found that antibiotics changed the neonatal microbiome from that seen among offspring that were not exposed to antibiotics and, importantly, the microbiota was altered even with short exposure to antibiotics, that is, not continued exposure throughout early life. ${ }^{17}$ Several studies have looked at the effect of intrapartum antibiotics on the neonatal microbiome. Corvaglia et al collected fecal samples of 84 infants on days 7 and 30 of life and found that there was significant difference in certain colonies among infants born from GBS-positive women who had received intrapartum antibiotics $(N=35)$ compared with infants born from GBSnegative women who did not receive intrapartum antibiotics $(N=49)$ at day 7 , but the difference diminished by day $30 .{ }^{18}$ The limitation of this study was that they only evaluated for three bacteria that may play an important role in the development of the microbiome. Another work, evaluating 52 newborns by fecal sampling on day 7, had a similar finding, but did not repeat fecal sampling at a later age. ${ }^{19}$ Finally, Mazzola et al evaluated the entire microbiome diversity on fecal samples using whole genome sequencing and polymerase chain reaction from 26 infants on days 7 and 30 of life. They found that infants who had exposure to intrapartum antibiotics in GBS-positive women had significantly lower diversity of bacteria compared with GBS-negative women on day 7 with only partial recovery on day $30 .{ }^{20}$ Whether the difference in diversity of the neonatal microbiome persists is currently unknown. ${ }^{21}$

Several other consequences of antibiotic exposure have been studied. Ahmadizar et al, in a meta-analysis, demonstrated an increased risk of asthma with antibiotic use in early life. They analyzed two large population-based cohorts from the Netherlands and Scotland, including 7,393 and 891 children, respectively, and found an increased risk of asthma with an odds ratio of 2.18 in children who had antibiotic exposure in the first 3 years of life. ${ }^{22}$ Another recent retrospective cohort revealed an association of antibiotic exposure in the first year of life and the development of asthma. Yoshida et al used health insurance claim data in Japan and found that antibiotic exposure during pregnancy was also associated with asthma development (hazards ratio: $1.18,95 \%$ confidence interval $[\mathrm{CI}]: 1.08-1.30$ ), though this association was only seen up to 3 years of age. ${ }^{23}$ In addition, a case-control study of 134 children with asthma demonstrated that antibiotic exposure during pregnancy was a significant risk factor for development of asthma (adjusted odds ratio: $3.19,95 \% \mathrm{CI}$ : $1.52-6.67){ }^{24}$

The association of eczema and prenatal antibiotic exposure has also been explored. Dom et al retrospectively analyzed a prospective cohort study of pregnant women who completed a questionnaire on antibiotic use during pregnancy, and assessed the subsequent occurrence of allergies, asthma, and eczema in their children up to 4 years of age. In the 773 children analyzed, they found an association of prenatal antibiotic exposure with development of eczema (adjusted odds ratio: $1.82,95 \% \mathrm{CI}: 1.14-2.92) .{ }^{25}$ Sariachvili et al analyzed 976 children from the same cohort to determine if breastfeeding had a protective effect on development of eczema within the first year of life, but they did not find a significant association. They did demonstrate an increased risk of eczema after antibiotic use in pregnancy (adjusted odds ratio: 1.8, 95\% CI: 1.2-2.7). ${ }^{26}$ However, a recent meta-analysis on the development of eczema and prenatal antibiotic exposure that included four observational studies found no significant difference in the risk of developing eczema with prenatal antibiotic exposure (odds ratio: $1.30,95 \% \mathrm{CI}: 0.86-1.95){ }^{27}$

There is also literature on the relationship of early antibiotic exposure and development of childhood obesity. Using longitudinal data, Mueller et al demonstrated an increased risk of childhood obesity at 7 years after prenatal exposure to antibiotics in the third trimester. They analyzed 436 motherchild dyads after collecting data on prenatal antibiotic use by using a questionnaire that was administered in the third trimester and analyzing data on actual body weight and 
measurement of the child at age 7. They found an increased risk of obesity associated with prenatal antibiotic exposure with an adjusted odds ratio: 1.77 (95\% CI: 1.25-2.51). They also found an association with cesarean delivery (adjusted odds ratio: $1.41,95 \% \mathrm{CI}: 1.01-1.96) .{ }^{28}$ It has been suggested that intestinal microbiota plays an essential role in these findings, and differences in the microbiome may lead to obesity. However, it is important to note that although there are statistical significance among the stated studies, the CIs are narrow and close to one, and may signal noise rather than harm. ${ }^{29}$ Nonetheless, it is imperative for us to be aware and prospectively assess and confirm this association. Antibiotic exposure is one of three key factors that may influence the neonate's microbiota, the other two being breastfeeding and mode of delivery. ${ }^{15}$ Currently, there are no direct studies evaluating the effect of intrapartum antibiotic exposure on childhood obesity; however, further information about this link should be forthcoming from ongoing research. For example, a group in Canada is currently recruiting low-risk women from a midwife practice, planning for vaginal birth after 37 weeks, with a planned study population of 240 . They will prospectively follow this cohort and obtain stool samples up to 3 years of age to describe the intestinal microbiome of infants who were breastfed, and to determine if infants born to women who receive intrapartum antibiotics for GBS or antibiotic prophylaxis have intestinal microbiota at the first year of life that differs significantly in type from those not exposed to antibiotics. ${ }^{30}$ This issue is concerning not only because of the direct medical harms from obesity but also because of the greater health care costs that will be incurred. One group of authors estimated that $\$ 190$ billion per year of health care spending is due to treating obesity and obesity-related conditions. $^{31}$

\section{Future Considerations and Solutions}

It must be noted that these studies assessed exposures that were usually much greater in magnitude than a single dose of antibiotics around the time of birth, and the risks associated with the lesser doses may be de minimis. On the contrary, there is some worrisome data associated with GBS prophylaxis, and it has been shown that it does not take prolonged exposure to antibiotics to alter the microbiome. If the studies on the microbiome, as well as the cited work on asthma and obesity, are borne out, then future investigations of antibiotics in pregnancy will need to weigh the benefits to the mother against potential harms to the child. This would be particularly important when the maternal benefits are marginal. A hypothetical example would be a study that showed that the number needed to treat to prevent one case of endometritis was 100 . While that one woman might be spared a prolonged course of antibiotics, and rarely, a more serious consequence, then it would be reasonable to ask, what biologic cost is borne by the 100 exposed neonates? To address this conundrum, we suggest two possible solutions. First, future studies on antibiotic prophylaxis should look at long-term effects on the neonates, until at least 3 years of age, to evaluate for outcomes such as obesity and asthma. In one study, bacterial species were characterized from fecal samples over the first year of life in 110 healthy children and were found to be similar to adult populations by the time the children reached the age of $3 .^{32}$ Thus, a straightforward outcome to consider will be the body mass index of children at the age of 3 . However, this outcome (as well as others such as asthma and eczema) may have confounding variables that were not addressed in the previous studies, including social determinant factors, such as type of housing and food security. It is important that these variables are accounted for when evaluating these outcomes in future studies. Though these studies will be difficult and logistically challenging, they are essential, if we are to be able to consider the development of chronic diseases when crafting recommendations for antibiotic use in pregnancy.

Second, there should be more studies looking at antibiotic prophylaxis after cord clamping to see if they can be made to have an efficacy similar to that seen with antibiotics given before clamping. A recent single site cohort study analyzed this issue, and found no difference in surgical site infections after cesarean sections after a policy change from antibiotic prophylaxis administered after cord clamping to preincision. ${ }^{33}$ In addition, Valent et al demonstrated in a randomized controlled trial that 48 hours of cephalexin and metronidazole in addition to the usual practice of preincision cefazolin administration reduced surgical site infections from 15 to $6 \%$ in obese women, which was a similar risk reduction from 12 to $6.1 \%$ seen in the trial by Tita et al.,34 If antibiotic prophylaxis after cord clamping in conjunction with postoperative antibiotic regimen were as effective as preincision antibiotic prophylaxis, this would dramatically reduce the exposure of antibiotics to the fetus and reduce their long-term effect.

In conclusion, we are not arguing that appropriate antibiotic prophylaxis should no longer be a standard of care in obstetrics. However, there is a need for investigators to recognize the current evidence based on observational studies. Therefore, the long-term effects of antibiotic exposure in the fetus/neonate (e.g., potential risk of asthma and childhood obesity related to changes in neonatal microbiota) should be considered when planning large-scale trials that will involve antibiotic prophylaxis.

\section{Conflict of Interest}

None declared.

\section{References}

1 Boyle A, Reddy UM. Epidemiology of cesarean delivery: the scope of the problem. Semin Perinatol 2012;36(05):308-314

2 Costantine MM, Rahman M, Ghulmiyah L, et al. Timing of perioperative antibiotics for cesarean delivery: a metaanalysis. Am J Obstet Gynecol 2008;199(03):301.e1-301.e6

3 Conroy K, Koenig AF, Yu YH, Courtney A, Lee HJ, Norwitz ER. Infectious morbidity after cesarean delivery: 10 strategies to reduce risk. Rev Obstet Gynecol 2012;5(02):69-77

4 Scifres CM, Leighton BL, Fogertey PJ, Macones GA, Stamilio DM. Supplemental oxygen for the prevention of postcesarean infectious morbidity: a randomized controlled trial. Am J Obstet Gynecol 2011;205(03):267.e1-267.e9

5 Wloch C, Wilson J, Lamagni T, Harrington P, Charlett A, Sheridan E. Risk factors for surgical site infection following caesarean section 
in England: results from a multicentre cohort study. BJOG 2012; 119(11):1324-1333

6 Tita AT, Szychowski JM, Boggess K, et al; C/SOAP Trial Consortium. Adjunctive azithromycin prophylaxis for cesarean delivery. N Engl J Med 2016;375(13):1231-1241

7 Caissutti C, Saccone G, Zullo F, et al. Vaginal cleansing before cesarean delivery: a systematic review and meta-analysis. Obstet Gynecol 2017;130(03):527-538

8 La Rosa M, Jauk V, Saade G, et al; Cesarean Section Optimal Antibiotic Prophylaxis Trial Consortium. Institutional protocols for vaginal preparation with antiseptic solution and surgical site infection rate in women undergoing cesarean delivery during labor. Obstet Gynecol 2018;132(02):371-376

9 Harper LM, Kilgore M, Szychowski JM, Andrews WW, Tita ATN. Economic evaluation of adjunctive azithromycin prophylaxis for cesarean delivery. Obstet Gynecol 2017;130(02):328-334

10 Blaser MJ. Our missing microbes: short-term antibiotic courses have long-term consequences. Cleve Clin J Med 2018;85(12):928-930

11 Rodriguez J, Jordan S, Mutic A, Thul T. The neonatal microbiome: implications for neonatal intensive care unit nurses. MCN Am J Matern Child Nurs 2017;42(06):332-337

12 Leis JA, Soong C. De-adoption of routine urine culture testing-a call to action. JAMA Intern Med 2019

13 Puopolo KM, Lynfield R, Cummings JJ; COMMITTEE ON FETUS AND NEWBORN; COMMITTEE ON INFECTIOUS DISEASES. Management of infants at risk for group B streptococcal disease. Pediatrics 2019;144(02):e20191881

14 Knight M, Chiocchia V, Partlett C, et al; ANODE collaborative group. Prophylactic antibiotics in the prevention of infection after operative vaginal delivery (ANODE): a multicentre randomised controlled trial. Lancet 2019;393(10189):2395-2403

15 Tamburini S, Shen N, Wu HC, Clemente JC. The microbiome in early life: implications for health outcomes. Nat Med 2016;22 (07):713-722

16 Jiménez E, Marín ML, Martín R, et al. Is meconium from healthy newborns actually sterile? Res Microbiol 2008;159(03):187-193

17 Cox LM, Yamanishi S, Sohn J, et al. Altering the intestinal microbiota during a critical developmental window has lasting metabolic consequences. Cell 2014;158(04):705-721

18 Corvaglia L, Tonti G, Martini S, et al. Influence of intrapartum antibiotic prophylaxis for group B Streptococcus on gut microbiota in the first month of life. J Pediatr Gastroenterol Nutr 2016; 62(02):304-308

19 Aloisio I, Mazzola G, Corvaglia LT, et al. Influence of intrapartum antibiotic prophylaxis against group B Streptococcus on the early newborn gut composition and evaluation of the anti-Streptococcus activity of Bifidobacterium strains. Appl Microbiol Biotechnol 2014;98(13):6051-6060

20 Mazzola G, Murphy K, Ross RP, et al. Early gut microbiota perturbations following intrapartum antibiotic prophylaxis to prevent group B streptococcal disease. PLoS One 2016;11(06): e0157527

21 Zimmermann P, Curtis N. Effect of intrapartum antibiotics on the intestinal microbiota of infants: a systematic review. Arch Dis Child Fetal Neonatal Ed 2020;105(02):201-208

22 Ahmadizar F, Vijverberg SJH, Arets HGM, et al. Early life antibiotic use and the risk of asthma and asthma exacerbations in children. Pediatr Allergy Immunol 2017;28(05):430-437

23 Yoshida S, Ide K, Takeuchi M, Kawakami K. Prenatal and early-life antibiotic use and risk of childhood asthma: a retrospective cohort study. Pediatr Allergy Immunol 2018;29(05):490-495

24 Kashanian M, Mohtashami SS, Bemanian MH, Moosavi SAJ, Moradi Lakeh M. Evaluation of the associations between childhood asthma and prenatal and perinatal factors. Int J Gynaecol Obstet 2017;137(03):290-294

25 Dom S, Droste JH, Sariachvili MA, et al. Pre- and post-natal exposure to antibiotics and the development of eczema, recurrent wheezing and atopic sensitization in children up to the age of 4 years. Clin Exp Allergy 2010;40(09):1378-1387

26 Sariachvili M, Droste J, Dom S, et al. Is breast feeding a risk factor for eczema during the first year of life? Pediatr Allergy Immunol 2007;18(05):410-417

27 Tsakok T, McKeever TM, Yeo L, Flohr C. Does early life exposure to antibiotics increase the risk of eczema? A systematic review. Br J Dermatol 2013;169(05):983-991

28 Mueller NT, Whyatt R, Hoepner L, et al. Prenatal exposure to antibiotics, cesarean section and risk of childhood obesity. Int J Obes 2015;39(04):665-670

29 Grimes DA, Schulz KF. False alarms and pseudo-epidemics: the limitations of observational epidemiology. Obstet Gynecol 2012; 120(04):920-927

30 Simioni J, Hutton EK, Gunn E, et al. A comparison of intestinal microbiota in a population of low-risk infants exposed and not exposed to intrapartum antibiotics: the baby \& microbiota of the intestine cohort study protocol. BMC Pediatr 2016;16(01):183

31 Cawley J, Meyerhoefer C. The medical care costs of obesity: an instrumental variables approach. J Health Econ 2012;31(01): 219-230

32 Yatsunenko T, Rey FE, Manary MJ, et al. Human gut microbiome viewed across age and geography. Nature 2012;486 (7402):222-227

33 Ben Shoham A, Bar-Meir M, Isocovich A, Samueloff A, WienerWell Y. Timing of antibiotic prophylaxis in cesarean section: retrospective, difference-in-differences estimation of the effect on surgical-site-infection. J Matern Fetal Neonatal Med 2019;32 (05):804-808

34 Valent AM, DeArmond C, Houston JM, et al. Effect of post-cesarean delivery oral cephalexin and metronidazole on surgical site infection among obese women: a randomized clinical trial. JAMA 2017;318(11):1026-1034 